\title{
COOLING OF A LAYERED PLATE UNDER MIXED CONDITIONS
}

\author{
F.D. ZAMAN \\ King Fahd University of Petroleum and Minerals \\ Department of Mathematical Sciences \\ Dhahran 31261, Saudi Arabia \\ R. AL-KHAIRY \\ Dammam College of Girls \\ Department of Mathematics \\ Dammam, Saudi Arabia
}

(Received June, 1998; Revised November, 1999)

\begin{abstract}
We consider the temperature distribution in an infinite plate composed of two dissimilar materials. We suppose that half of the upper surface $(y=h,-\infty<x<0)$ satisfies the general boundary condition of the Neumann type, while the other half $(y=h, 0<x<\infty)$ satisfies the general boundary condition of the Dirichlet type. Such a plate is allowed to cool down on the lower surface with the help of a fluid medium which moves with a uniform speed $v$ and which cools the plate at rate $\Omega$. The resulting mixed boundary value problem is reduced to a functional equation of the Wiener-Hopf type by use of the Fourier transform. We then seek the solution using the analytic continuation and an extended form of the Liouville theorem. The temperature distribution in the two layers can then be written in a closed form by use of the inversion integral.
\end{abstract}

Key words: Heat Equation, Layered Plate, Mixed Boundary Conditions.

AMS subject classifications: $35 \mathrm{~K} 20,45 \mathrm{E} 10,80 \mathrm{~A} 20$.

\section{Introduction}

The problem of heat flow in layered and composite structures has attracted considerable attention in the last decades. The simplest problem is that of the one-dimensional heat conduction or linear heat flow. Carslaw and Jaeger [3] have discussed different aspects of linear heat flow in plates and rods having homogeneous or composite structures. In such problems, the boundary of the body under consideration is either assumed to be insulated or kept at a constant temperature. Some problems of practical interest, however, require imposition of mixed boundary conditions. The classical 
transform or Fourier series techniques are then no longer applicable. An ingenious method of dealing with such problems is the use of the Wiener-Hopf technique.

One of the early studies in this regard is by Caflisch and Keller [2] who have considered the problem of steady state heat conduction in a sufficiently hot plate being cooled by water flowing over half of its upper surface while the other half and its lower surface are kept insulated. The water adjacent to the hotter part is converted to steam, while the water adjacent to the cooler part remains in liquid form. This situation results in mixed boundary conditions as the part which is treated by the flowing liquid satisfies the cooling condition while the remaining part may be considered as being insulated. The solution in terms of an infinite product involving the roots of a certain transcendental equation is then obtained using the Wiener-Hopf technique. More details about this technique may be found in the treatise by Noble $[8]$.

Levine [7] also considered this problem, but assumed a simpler representation of the sputtering temperature. In both cases [2] and [7], the authors first obtained appropriate Green's functions for the problem. Thus, in each case, the problem was reduced to a singular integral equation which could then be solved by use of the Wiener-Hopf technique.

Evans [4] considered the problem of lowering a long circular cylinder at a uniform temperature into a cooling liquid. This liquid cools the lower half at a constant rate, while the upper half remains insulated. However, instead of reducing the resulting mixed boundary value problem to an integral equation, Evans used the modification due to Jones [6] which yields the so-called Wiener-Hopf functional equation without the need of a Green's function. Georgiadis, Barber and Ben Ammar [5], Bera and Chakrabarti [1] and Zaman [9] have considered mixed boundary value problems arising from layered media having either mixed interfaces or mixed cooling conditions using the Jones modified method.

In this paper, we study a more general model of the steady state cooling problem by assuming general mixed boundary conditions over the upper surface of an infinite rectangular plate composed of two materials with different thermal properties. We suppose that half of the upper surface satisfies one general boundary condition. Such a plate is allowed to cool with the help of a fluid medium which moves with a uniform speed $v$ and which affects a constant rate of cooling $\Omega$ on the lower surface of the plate. Using the Jones modification of the Wiener-Hopf technique, we transform this problem into the Wiener-Hopf equation and obtain the solution in a closed form.

\section{Formulation of the Problem}

We consider an infinite rectangular plate of uniform thickness $h$, composed of two homogeneous but dissimilar materials. The faces of the plate are represented by the planes $y=0$ and $y=h$. We write $K_{1}, k_{1}$ and $u_{1}(x, y)$ for the conductivity, the diffusivity and the steady state temperature distribution in the upper layer $0<y<\delta$; and the corresponding quantities in the lower layer $\delta<y<h$ are denoted by $K_{2}, k_{2}$ and $u_{2}(x, y)$, respectively.

The partial differential equations satisfied by $u_{i}(x, y), i=1,2$ are

$$
\nabla^{2} u_{1}=2 s_{1} \frac{\partial u_{1}}{\partial x}, 0<y<\delta
$$




$$
\nabla^{2} u_{2}=2 s_{2} \frac{\partial u_{2}}{\partial x}, \delta<y<h
$$

where

$$
\nabla^{2} \equiv \frac{\partial^{2}}{\partial x^{2}}+\frac{\partial^{2}}{\partial y^{2}}, s_{j}=\frac{v}{2 k_{j}}, \quad j=1,2 .
$$

We seek to solve equations (1) and (2) under the following set of conditions:

(i) For the lower surface,

$$
\frac{\partial u_{1}}{\partial y}+\Omega u_{1}=0 \text { on } y=0,-\infty<x<\infty
$$

(ii) For the interface

$$
\left.\begin{array}{rl}
u_{1} & =u_{2} \\
K_{1} \frac{\partial u_{1}}{\partial y} & =K_{2} \frac{\partial u_{2}}{\partial y}
\end{array}\right\} \text { on } y=\delta, \quad-\infty<x<\infty
$$

(iii) For the other surface,

$$
\begin{aligned}
& u_{2}(x, h)=f(x) \text { on } y=h, 0 \leq x<\infty \\
& \frac{\partial u_{2}}{\partial y}=g(x) \text { on } y=h, \quad-\infty<x<0 .
\end{aligned}
$$

(iv) In addition, we assume that there is a particular level of temperature difference between the extremities of the composite plate, i.e.,

$$
\begin{gathered}
u_{1}, u_{2} \rightarrow 1 \text { as } x \rightarrow \infty, \\
u_{1}, u_{2} \rightarrow 0 \text { as } x \rightarrow-\infty
\end{gathered}
$$

and

$$
u_{2}, \frac{\partial u_{2}}{\partial y} \sim O(1) \text { as } x \rightarrow 0
$$

Moreover,

$$
\begin{gathered}
|f(x)|<c_{1} \exp \left(\tau_{-} x\right) \text { as } x \rightarrow \infty \\
|g(x)|<c_{2} \exp \left(\tau_{+} x\right) \text { as } x \rightarrow-\infty
\end{gathered}
$$

where $c_{1}, c_{2}, \tau_{+}$and $\tau_{-}$are constants and $-s<\tau_{-}<\tau_{+}<s$ where $s=\min \left\{s_{1}, s_{2}\right\}$.

We shall use the Jones method to reduce the above mixed boundary value problem to the Wiener-Hopf equation. When we set

$$
u_{j}(x, y)=\exp \left(s_{j} x\right) \phi_{j}(x, y), \quad j=1,2
$$

equations (1) and (2) reduce to 


$$
\nabla^{2} \phi_{j}-s_{j}^{2} \phi_{j}=0, \quad j=1,2 .
$$

The boundary and the interface conditions along the parallel sides now become

$$
\begin{gathered}
\frac{\partial \phi_{1}}{\partial y}+\Omega \phi_{1}=0 \text { on } y=0,-\infty<x<\infty, \\
\exp \left(s_{1} x\right) \phi_{1}=\exp \left(s_{2} x\right) \phi_{2} \\
\left.K_{1} \exp \left(s_{1} x\right) \frac{\partial \phi_{1}}{\partial y}=K_{2} \exp \left(s_{2} x\right) \frac{\partial \phi_{2}}{\partial y}\right\} \text { on } y=\delta,-\infty<x<\infty, \\
\phi_{2}(x, y)=f(x) \exp \left(-s_{2} x\right) \text { on } y=h,-\infty<x<0, \\
\frac{\partial \phi_{2}}{\partial y}=g(x) \exp \left(-s_{2} x\right) \text { on } y=h,-\infty<x<0 .
\end{gathered}
$$

The behavior of the solution of the extremities is governed by

$$
\left.\begin{array}{c}
\phi_{1} \sim \epsilon \exp \left(-s_{1} x\right) \rightarrow 0, \phi_{2} \sim \epsilon \exp \left(-s_{2} x\right) \rightarrow 0, \text { as } x \rightarrow-\infty \\
\phi_{1} \sim \exp \left(-s_{1} x\right), \phi_{2} \sim \exp \left(-s_{2} x\right), \text { as } x \rightarrow \infty
\end{array}\right\}
$$

where $\epsilon$ is an arbitrary small number. Moreover, the temperature should be bounded at $x=0$ on $y=h$, so that

$$
\phi_{2}, \frac{\partial \phi_{2}}{\partial y} \sim O(1)
$$

\section{Reduction to the Wiener-Hopf Equation}

We define the Fourier transform in $x$ and its inverse as

$$
f^{*}(\alpha)=\int_{-\infty}^{\infty} f(x) \exp (i \alpha x) d x
$$

and

$$
f(x)=\frac{1}{2 \pi} \int_{-\infty+i d}^{\infty+i d} f^{*}(\alpha) \exp (-i \alpha x) d \alpha
$$

where $d$ is a constant chosen in the domain of analyticity of $f^{*}(\alpha)$ which is the strip $\tau_{-} \leq \operatorname{Im}(\alpha) \leq \tau_{+}$.

The half-range Fourier transforms are defined by

$$
f_{+}^{*}(\alpha)=\int_{0}^{\infty} f(x) \exp (i \alpha x) d x
$$




$$
f_{-}^{*}(\alpha)=\int_{-\infty}^{0} f(x) \exp (i \alpha x) d x
$$

so that

$$
f^{*}(\alpha)=f_{+}^{*}(\alpha)+f_{-}^{*}(\alpha) .
$$

It may be noted that if $f(x)=O\left(e^{\tau-x}\right)$ as $x \rightarrow \infty$ and $f(x)=O\left(e^{\tau}+{ }^{x}\right)$ as $x \rightarrow-\infty$, then $f_{+}^{*}(\alpha)$ is an analytic function of $\alpha$ in the upper half-plane $\operatorname{Im}(\alpha)>\tau_{-}$, while $f_{-}^{*}(\alpha)$ is an analytic function of $\alpha$ in the lower half-plane $\operatorname{Im}(\alpha)<\tau_{+}$. By virtue of equation $(24), f^{*}(\alpha)$ is then analytic in $\tau_{-} \leq \operatorname{Im}(\alpha) \leq \tau_{+}$ (Nobel [8]).

Applying the Fourier transform to the partial differential equations (13), we get

$$
\frac{d^{2} \phi_{j}^{*}(\alpha, y)}{d y^{2}}-\gamma_{j}^{2} \phi_{j}^{*}(\alpha, y)=0, \quad j=1,2
$$

where

$$
\gamma_{j}=\gamma_{j}(\alpha)=\sqrt{\alpha^{2}+s_{j}^{2}}
$$

The branches of $\gamma_{j}$ are chosen such that

$$
\gamma_{j}(0)=+s_{j}
$$

The boundary and the interface conditions are transformed into

$$
\left.\begin{array}{c}
\phi_{1}^{*^{\prime}}(\alpha, y)+\Omega \phi_{1}^{*}(\alpha, y)=0 \text { on } y=0 \\
\phi_{1}^{*}\left(\alpha-i s_{1}, y\right)=\phi_{2}^{*}\left(\alpha-i s_{2}, y\right) \\
K_{1} \phi_{1}^{*^{\prime}}\left(\alpha-i s_{1}, y\right)=K_{2} \phi_{2}^{*^{\prime}}\left(\alpha-i s_{2}, y\right)
\end{array}\right\} \text { on } y=\delta,
$$

The functions $\phi_{j}^{*+}(\alpha, y)$ and $\phi_{j}^{*^{\prime}+}(\alpha, y)$ are analytic in the region

$$
\operatorname{Im}(\alpha)>\max \left(-s_{1},-s_{2}\right) \equiv-s
$$

and the functions $\phi_{j}^{*-}(\alpha, y)$ and $\phi_{j}^{*^{\prime}-}(\alpha, y)$ are analytic in the region

$$
\operatorname{Im}(\alpha)<\min \left(s_{1}, s_{2}\right) \equiv s
$$

Thus the functions $\phi_{j}^{*}(\alpha, y)$ and $\phi_{j}^{*^{\prime}}(\alpha, y)$ are analytic in the common strip $-s<\operatorname{Im}(\alpha)<s$.

The solution to $(20)$ is given by

$$
\phi_{1}^{*}(\alpha, y)=A_{1}(\alpha) \cosh \gamma_{1} y+B_{1}(\alpha) \sinh \gamma_{1} y
$$


and

$$
\phi_{2}^{*}(\alpha, y)=B(\alpha) \cosh \gamma_{2} y+C(\alpha) \sinh \gamma_{2} y
$$

Applying the boundary and interface conditions (28)-(31), we obtain

$$
\phi_{2}^{*}(\alpha, h)=A\left(\alpha+i\left(s_{2}-s_{1}\right)\right) P\left[f_{1}(\alpha) \cosh \gamma_{2} h+f_{2}(\alpha) \frac{\sinh \gamma_{2} h}{\gamma_{2}}\right]
$$

and

$$
\phi_{2}^{*^{\prime}}(\alpha, h)=P\left[\gamma_{2} f_{1}(\alpha) \sinh \gamma_{2} h+f_{2}(\alpha) \cosh \gamma_{2} h\right] A\left(\alpha+i\left(s_{2}-s_{1}\right)\right),
$$

where

$$
\begin{gathered}
P\left(\alpha, s_{1}, s_{2}\right)=\sqrt{\left(\alpha+i s_{2}\right)\left(\alpha-2 i s_{1}+i s_{2}\right)} \\
f_{1}(\alpha)=\cosh \gamma_{2} \delta\left\{\cosh P \delta-\frac{\Omega \sinh P \delta}{P}\right\}-\frac{K_{1}}{K_{2} \gamma} \sinh \gamma_{2} \delta\{P \sinh P \delta-\Omega \cosh P \delta\}
\end{gathered}
$$

and

$$
\begin{gathered}
f_{2}(\alpha)=\frac{K_{1}}{K_{2}} \cosh \gamma_{2} \delta\{P \sinh P \delta-\Omega \cosh P \delta\} \\
-\gamma_{2} \sinh \gamma_{2} \delta\left\{\cosh P \delta-\frac{\Omega \sinh P \delta}{P}\right\} .
\end{gathered}
$$

In terms of $P$ and $f_{1}(\alpha)$ and $f_{2}(\alpha)$, we can obtain

$$
B(\alpha)=P A\left\{\alpha+i\left(s_{2}-s_{1}\right)\right\} f_{1}(\alpha)
$$

and

$$
C(\alpha)=\frac{P}{\gamma_{2}} A\left\{\alpha+i\left(s_{2}-s_{1}\right)\right\} f_{2}(\alpha) .
$$

Eliminating $A\left(\alpha+i\left(s_{2}-s_{1}\right)\right)$ from equations (36) and (37), we arrive at the Wiener-Hopf equation

$$
f_{+}^{*}\left(\alpha+i s_{2}\right)+\phi_{2}^{*-}(\alpha, h)=L(\alpha)\left[g_{-}^{*}\left(\alpha+i s_{2}\right)+\phi_{2}^{*^{\prime}}(\alpha, h)\right]
$$

where

$$
L(\alpha)=\frac{f_{1}(\alpha) \cosh \gamma_{2} h+f_{2}(\alpha) \frac{\sinh \gamma_{2} h}{\gamma_{2}}}{\gamma_{2} f_{1}(\alpha) \sinh \gamma_{2} h+f_{2}(\alpha) \cosh \gamma_{2} h}=\frac{G_{1}(\alpha, h)}{G_{2}(\alpha, h)}
$$

\section{Solution of the Wiener-Hopf Problem}

The forms of the function $f_{1}(\alpha)$ and $f_{2}(\alpha)$ as given by (39) and (40) suggest that the numerator and the denominator of $L(\alpha)$ do not have any branch points in the complex $\alpha$-plane. Thus $L(\alpha)$ can be written in the form 


$$
L(\alpha)=A \prod_{n=1}^{\infty}\left(\frac{\alpha^{2}+\alpha_{n}^{2}}{\alpha^{2}+\beta_{n}^{2}}\right), \alpha_{n}, \beta_{n}>0
$$

where

$$
A=\left(\prod_{n=1}^{\infty} \frac{\beta_{n}}{\alpha_{n}}\right)\left[\frac{f_{1}(0) \cosh s_{2} h+f_{2}(0) \frac{\sinh s_{2} h}{\gamma_{2}}}{s_{2} f_{1}(0) \sinh s_{2} h+f_{2}(0) \cosh s_{2} h}\right] .
$$

We can then write

$$
L^{+}(\alpha)=A^{1 / 2} \prod_{n=1}^{\infty}\left(\frac{\alpha+i \alpha_{n}}{\alpha+i \beta_{n}}\right)
$$

and

$$
L^{-}(\alpha)=A^{1 / 2} \prod_{n=1}^{\infty}\left(\frac{\alpha-i \alpha_{n}}{\alpha-i \beta_{n}}\right)
$$

where each $\alpha_{n}$ for $n=1,2, \ldots$ is a simple zero of the function $G_{1}(\alpha, h)$ and each $\beta_{n}$, for $n=1,2, \ldots$ is a simple zero of $G_{2}(\alpha, h)$. (These $\alpha$ 's and $\beta$ 's include all the simple zeros of $G_{1}(\alpha, h)$ and $G_{2}(\alpha, h)$.) $L^{+}(\alpha)$ is free from zeros and poles in the upper halfplane given by

$$
\tau=\operatorname{Im}(\alpha)>\max \left(-\alpha_{1},-\beta_{1}\right) \equiv \tau_{1-}
$$

and $L^{-}(\alpha)$ is free from zeros and poles in the lower half-plane given by

$$
\tau=\operatorname{Im}(\alpha)<\min \left(\alpha_{1}, \beta_{1}\right) \equiv \tau_{1+}
$$

Thus the Wiener-Hopf equation (43) becomes

$$
f_{+}^{*}\left(\alpha+i s_{2}\right)+\phi_{2}^{*-}(\alpha, h)=L^{+}(\alpha) L^{-}(\alpha)\left[g_{-}^{*}\left(\alpha+i s_{2}\right)+\phi_{2}^{*^{\prime}}(\alpha, h)\right] .
$$

Dividing by $L^{-}(\alpha)$ and rearranging, we get

$$
\frac{\phi_{2}^{*-}(\alpha)}{L^{-}(\alpha)}-H_{-}(\alpha)=L^{+}(\alpha) \phi_{2}^{*^{\prime}}(\alpha, h)+H_{+}(\alpha)
$$

where we have used the decomposition

$$
H(\alpha)=H_{+}(\alpha)+H_{-}(\alpha)=L^{+}(\alpha) g_{-}^{*}\left(\alpha+i s_{2}\right)-\frac{f_{+}^{*}\left(\alpha+i s_{2}\right)}{L^{-}(\alpha)},
$$

for $\tau_{-}<c<c^{\prime}<\tau_{+}$. Here $H_{+}(\alpha)$ and $H_{-}(\alpha)$ are given by the factorization theorem (Noble [8]) as

$$
H_{ \pm}(\alpha)=\frac{ \pm 1}{2 \pi i} \int_{-\infty+i c^{\prime}}^{\infty+i c^{\prime}}\left[L^{+}(\eta) g_{-}^{*}\left(\eta+i s_{2}\right)-\frac{f_{+}^{*}\left(\eta+i s_{2}\right)}{L^{-}(\eta)}\right] \frac{d \eta}{\eta-\alpha}
$$

We note that $H_{+}(\alpha)$ is analytic in $\tau>c$ and $H_{-}(\alpha)$ is analytic in $\tau<c^{\prime}$. The left-hand side of equation (52) is an analytic function on the lower half-plane $\operatorname{Im}(\alpha)<d_{+}$, while the right-hand side is an analytic function on the upper half-plane $\operatorname{Im}(\alpha)>d_{-}$where the strip $d_{-}<\operatorname{Im}(\alpha)<d_{+}$, is the smallest common strip of 
analyticity between all the + and - functions. Due to this strip, both sides define an entire function of $\alpha$, say, $J(\alpha)$ by analytic continuation, which has algebraic behavior as $\alpha \rightarrow \infty$. We can use the extended form of the Liouville theorem to determine the exact form of this analytic function $J(\alpha)$. From (10), (11), (18), (19) and the abelian theorems on Fourier transforms concerning asymptotic relations between functions and their Fourier transforms in conjunction with the asymptotic behavior of infinite products as $\alpha \rightarrow \infty$ (see Noble [8], p. 128), it can be proved that $J(\alpha) \rightarrow 0$ as $\alpha \rightarrow \infty$ in any direction in the $\alpha$-plane. Hence, from the Liouville theorem, $J(\alpha)$ is identically zero. Thus

$$
\phi_{2}^{*-}(\alpha, h)=L^{-}(\alpha) H_{-}(\alpha)
$$

and

$$
\phi_{2}^{*^{\prime}}(\alpha, h)=\frac{H+(\alpha)}{L^{+}(\alpha)}
$$

By use of residue calculus, the explicit forms of $H_{+}(\alpha)$ and $H_{-}(\alpha)$ can be obtained from equation (54) as follows:

$$
\begin{gathered}
H_{-}(\alpha)=L^{+}(\alpha) g_{-}^{*}\left(\alpha+i s_{2}\right)-\sum_{j=1}^{\infty} \frac{a_{j}^{*} g_{-}^{*}\left(-i \beta_{j}+i s_{2}\right)}{-i \beta_{j}-\alpha} \\
-\sum_{j=1}^{\infty} a_{j} \frac{f_{+}^{*}\left(i \alpha_{j}+i s_{2}\right)}{i \alpha_{j}-\alpha}
\end{gathered}
$$

and

$$
H_{+}(\alpha)=-\frac{f_{+}^{*}\left(\alpha+i s_{2}\right)}{L^{-}(\alpha)}+\sum_{j=1}^{\infty} \frac{a_{j}^{*} g_{-}^{*}\left(-i \beta_{j}+i s_{2}\right)}{-i \beta_{j}-\alpha}+\sum_{j=1}^{\infty} a_{j} \frac{f_{+}^{*}\left(i \alpha_{j}+i s_{2}\right)}{i \alpha_{j}-\alpha}
$$

where

$$
a_{j}^{*}=A^{1 / 2} i\left(\beta_{j}-\alpha_{j}\right) \prod_{n=1, n \neq j}^{\infty}\left(\frac{\beta_{j}-\alpha_{n}}{\beta_{j}-\beta_{n}}\right)
$$

and

$$
a_{j}=A^{1 / 2} i\left(\alpha_{j}-\beta_{j}\right) \prod_{n=1, n \neq j}^{\infty}\left(\frac{\alpha_{j}-\beta_{n}}{\alpha_{j}-\alpha_{n}}\right)
$$

From equation (36), we get

$$
A\left(\alpha+i\left(s_{2}-s_{1}\right)\right)=\frac{f_{+}^{*}\left(\alpha+i s_{2}\right)+\phi_{2}^{*}-(\alpha, h)}{P G_{1}(\alpha, h)} .
$$

Similarly, from equation (37), we get

$$
A\left(\alpha+i\left(s_{2}-s_{1}\right)\right)=\frac{G_{-}^{*}\left(\alpha+i s_{2}\right)+\phi_{2}^{*^{\prime}+}(\alpha, h)}{P G_{2}(\alpha, h)} .
$$

The values of $B(\alpha)$ and $C(\alpha)$ can now be evaluated using equations (41) and (42). The solution in the transformed plane can thus be written as 


$$
\phi_{1}^{*}(\alpha, y)=\left[\frac{f_{+}^{*}\left(\alpha+i s_{1}\right)+\phi_{2}^{*}-\left(\alpha-i\left(s_{2}-s_{1}\right), h\right)}{G_{1}\left(\alpha-i\left(s_{2}-s_{1}\right), h\right)}\right]\left[\cosh \gamma_{1} y-\frac{\Omega \sinh \gamma_{1} y}{\gamma_{1}}\right]
$$

and

$$
\begin{gathered}
\phi_{2}^{*}(\alpha, y)=\left[\frac{f_{+}^{*}\left(\alpha+i s_{2}\right)+\phi_{2}^{*-}(\alpha, h)}{G_{1}(\alpha, h)}\right]\left[f_{1}(\alpha) \cosh \gamma_{2} y+f_{2}(\alpha) \frac{\sinh \gamma_{2} y}{\gamma_{2}}\right] \\
=\left[f_{+}^{*}\left(\alpha+i s_{2}\right)+\phi_{2}^{*-}(\alpha, h)\right] \frac{G_{1}(\alpha, y)}{G_{1}(\alpha, h)}
\end{gathered}
$$

It can be verified that (63) and (64) satisfy the boundary and the interface conditions (28)-(31).

\section{Fourier Transform Inversion}

Up to this point of analysis, we have determined the solution of the heat problem in the transformed $\alpha$-plane. In order to get the solution in the original $(x, y)$-plane, we should take the Fourier inverse transforms of equations (63) and (64) as follows:

$$
\begin{aligned}
\phi_{1}(x, y) & =\frac{1}{2 \pi} \int_{-\infty}^{\infty}\left[f_{1}^{*}\left(\alpha+i s_{1}\right)+\phi_{2}^{*-}\left(\alpha-i\left(s_{2}-s_{1}\right), h\right)\right] \\
& {\left[\frac{\cosh \gamma_{1} y-\frac{\Omega \sinh \gamma_{1} y}{\gamma_{1}}}{G_{1}\left(\alpha-i\left(s_{2}-s_{1}\right), h\right)}\right] \exp (-i \alpha x) d \alpha }
\end{aligned}
$$

and

$$
\phi_{2}(x, y)=\frac{1}{2 \pi} \int_{-\infty}^{\infty}\left[f_{+}^{*}\left(\alpha+i s_{2}\right)+\phi_{2}^{*-}(\alpha, h)\right] \frac{G_{1}(\alpha, y)}{G_{1}(\alpha, h)} \exp (-i \alpha x) d \alpha .
$$

We note that $\cosh \gamma_{1} y-\frac{\Omega \sinh \gamma_{1} y}{\gamma_{1}}$ and $G_{1}(\alpha, h)$ do not have any branch points in the complex $\alpha$-plane. We can use this fact and the residue theorem to obtain an infinite series representation for each $\phi_{1}(x, y)$ and $\phi_{2}(x, y)$, once specific forms of boundary functions of $f(x)$ and $g(x)$ are known. As an example of practical interest, one may put $g(x)=0$, which would correspond to the case of a plate which is insulated on the left half of the upper surface while kept at a prescribed temperature $f(x)$ on the right half of the upper surface. Other cases of interest can similarly be recovered from equations (65) and (66).

\section{Acknowledgment}

The first author wishes to acknowledge the support provided by the King Fahd University of Petroleum and Minerals. 


\section{References}

[1] Bera, R.K. and Chakrabarti, A., Cooling of a composite slab in two-fluid medium, ZAMP 42 (1991), 943-959.

[2] Caflisch, R.E. and Keller, J.B., Quench front propagation, Nuclear Eng. E Design 65 (1981), 97-102.

[3] Carslaw, H. and Jaeger, J.C., The Conduction of Heat in Solids, 2nd edition, Clarendon Press, Oxford 1959.

[4] Evans, D.V., A note on the cooling of a cylinder entering a fluid, IMA J. of Appl. Math. 33 (1984), 49-54.

[5] Georgiadis, H.G., Barber, J.R. and Ammar, F. Ben, An asymptotic solution for short time transient heat conduction between two dissimilar bodies in contact, Quart. J. Math. 44:2 (1991), 303-332.

[6] Jones, D.S., A simplifying technique, in the solution of a class of diffraction problems, Quart. J. Math. 2:3 (1952), 189-196.

[7] Levine, H., On a mixed boundary value problem of diffusion type, Applied Sci. Res. 39 (1982), 261-276.

[8] Noble, B., Methods Based on the Wiener-Hopf Technique, Pergamon Press, New York 1958.

[9] Zaman, F.D., Heat conduction across a semi-infinite interface in a layered plate, Utilitas Mathematica 43 (1993), 89-96. 


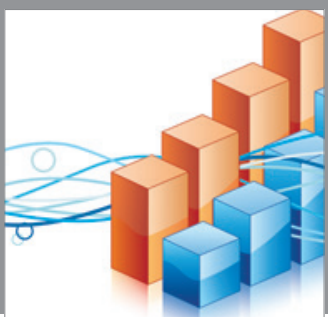

Advances in

Operations Research

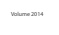

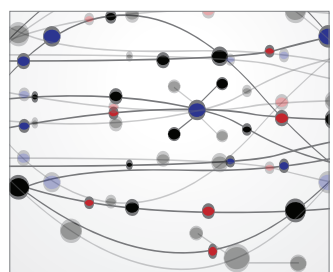

\section{The Scientific} World Journal
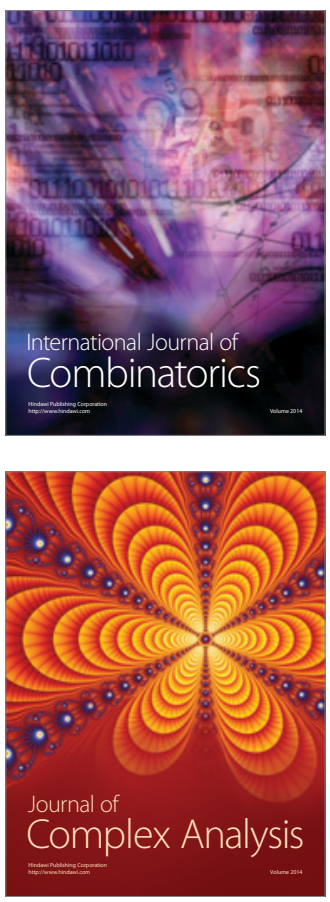

International Journal of

Mathematics and

Mathematical

Sciences
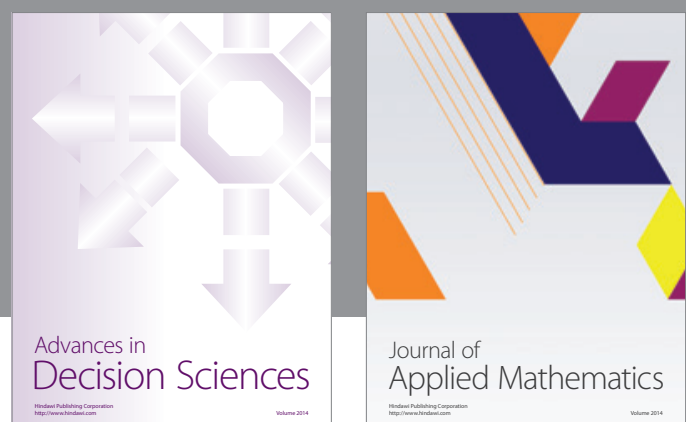

Journal of

Applied Mathematics
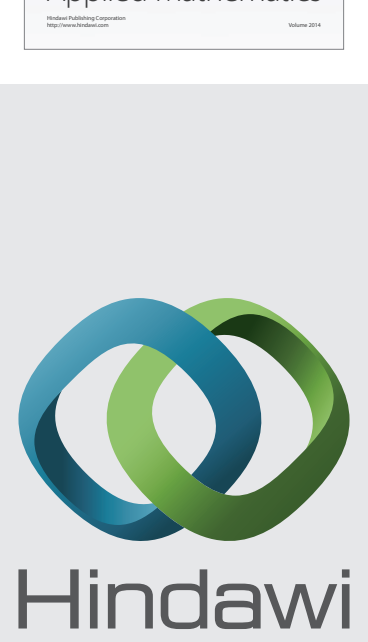

Submit your manuscripts at http://www.hindawi.com
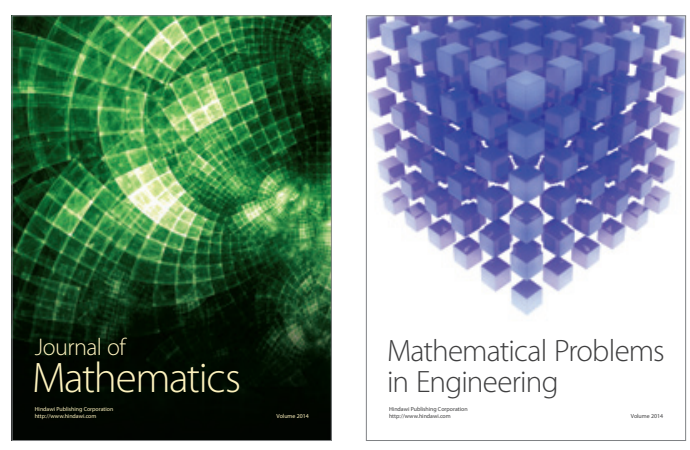

Mathematical Problems in Engineering
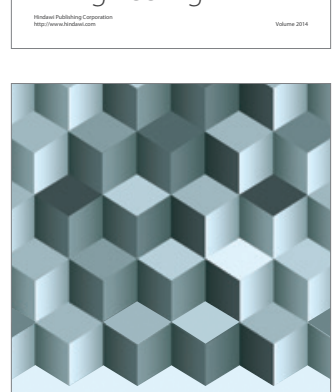

Journal of

Function Spaces
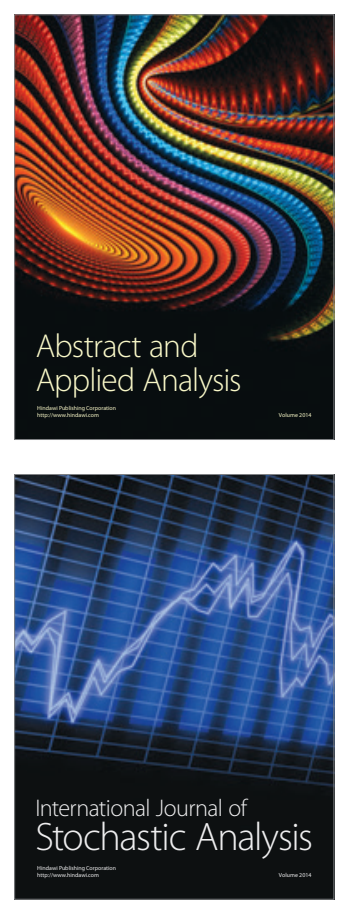

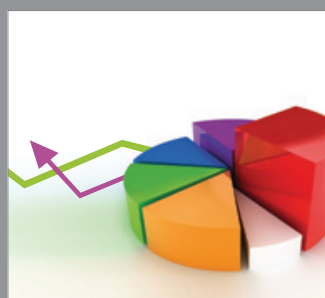

ournal of

Probability and Statistics

Promensencen
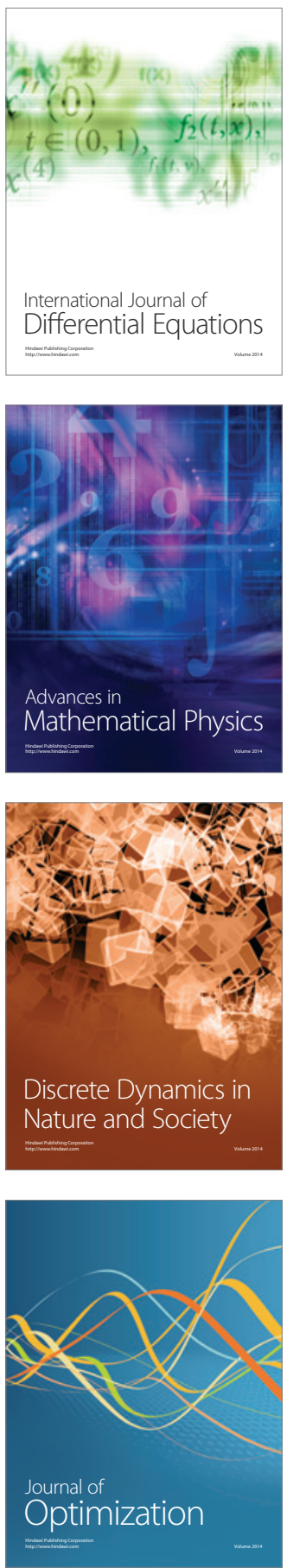